\title{
Editorial
}

\section{Veinte años buscando identidad}

Estimados(as) lectores(as),

No siempre se llega a 20 años de trabajo editorial. Hoy es para nosotros motivo de gran alegría presentar nuestro volumen XX, el cual es el resultado del proceso social de una comunidad que ha venido luchando y posicionándose, integrada por la Facultad de Ciencias Económicas de la Universidad Militar Nueva Granada, junto a sus amigos y amigas, y colaboradores todos. Docentes, autores(as), evaluadores(as), miembros de comités, asesores y personal técnico, son quienes han hecho posible que nuestras páginas se vuelvan un reflejo tanto de sus inquietudes, como de la realidad económica y social de nuestro país.

La Revista es un proyecto inacabado, pero esto, más que un problema, hace parte de su personalidad, siempre ha sido así, ya que desde su inicio ha tenido que responder a diversas exigencias. Nuestra publicación es joven, hoja de una Facultad que ha luchado por construir reconocimiento y legitimidad en un contexto donde diversas escuelas han tenido tradición, y por ello ventaja. La experimentación, incluso la improvisación ha dado paso a la tecnificación y profesionalización de nuestra labor. Ser un medio de comunicación económica con características científicas es un reto cada día más difícil. Cuando revisamos nuestras páginas, encontramos que no hemos terminado de recorrer el camino, por el contrario, cada vez los senderos nos advierten que, existen desafíos a futuro que requieren de ese elemento en el que se ha venido trabajando, es decir, en construir comunidad.

El presente editorial, es quizás el más ambicioso que hemos elaborado, porque en unas pocas cuartillas pretende sintetizar (quizás sin éxito), nuestra historia reciente, con la de los esfuerzos realizados por nuestros autores.

\section{El periodo 1992-2012 en nuestra economía, 20 volúmenes de discusiones económicas en nuestras páginas}

Terminado la década de los ochenta y en el marco del gobierno de Virgilio Barco Vargas la historia política y económica de nuestro país empezaba a dar un giro significativo. Bajo los últimos aires del modelo de sustitución de importaciones (ISI), y dando pasos importantes hacia al comercio exterior, la sociedad colombiana conocía nuevas formas de violencia ligadas, ya no solo a la lucha entre el Estado y movimientos alzados en armas, sino a la configuración de nuevas actores que con el apoyo de facciones de la sociedad más conservadoras y con dineros provenientes del narcotráfico, generaron una nueva 
cotidianidad. Días turbulentos afrontaron los colombianos de ese entonces, lo que llevo a grados de intimidación que forzaron a cambios fundamentales en lo que se reconoce como la historia reciente de Colombia.

En ese marco, pesimista y confuso aparece el Volumen I (1) de nuestra publicación. En ese entonces, la Revista concebida como mecanismo de comunicación de una naciente Facultad integro 9 artículos, 3 de ellos de autores anónimos. No se trataba de una revista científica, más bien era el esfuerzo de algunos docentes, directivos y amigos para exponer algunas reflexiones sobre su quehacer, o sobre inquietudes de la realidad nacional. Temas como el comercio internacional (Garzón, 1986), la seguridad alimentaria (Rodríguez, 1986), o la administración pública (Reyes, 1986), entre otros, fueron los motivos de reflexión de este ejemplar. Publicado en tamaño media carta, además de las colaboraciones de los articulistas, los primeros ejemplares presentaban un calendario académico, una carátula en la que cuatro diagramas eran el elemento central sobre una cuadrícula bicolor y una foto de la recientemente inaugurada sede de la calle 100.

La década de los noventa inicia, y con ella la consolidación de una serie de cambios importantes. Luego de la reñida (más por las posiciones políticas que se manifestaron) y sangrienta campaña presidencial (asesinato de los candidatos presidenciales Bernardo Jaramillo Ossa, Carlos Pizarro y Luis Carlos Galán, de los lideres políticos Jaime Pardo Leal y José Antequera, el atentado contra Ernesto Samper Pizano y el secuestro de Álvaro Gómez Hurtado, entre otros) en el marco de una guerra desmedida, llega a la presidencia de la república Cesar Gaviria Trujillo, quien inicia una serie de reformas importantes. Retomando los esfuerzos del Movimiento de la séptima papeleta se convoca y adelanta una Asamblea Nacional Constituyente, que consolidó la Carta Política que vino a delinear la estructura del país desde entonces, hasta nuestros días. La nueva constitución significo una revolución importante en el tipo de país, dio paso a un Estado descentralizado, laico, multicultural y moderno, garante de derechos en lo político. Pero también significo el paso a un nuevo modelo económico, neoliberal, flexible, aperturista, en el que el Estado cedió casi por completo el control del mercado financiero, laboral y de capitales. Más derechos y más mercado, no siempre es la combinación óptima, pero como estrategia ha delineado el pasado y presente reciente de los colombianos. Sin ser la panacea, el nuevo estatuto social saco del ostracismo centralizador y del oscurantismo a la nación, dejando atrás el pacto enmarcado en la constitución de 1886 y tratando de superar los vicios creados con el Frente Nacional.

No es clara la razón, pero el Volumen II (1) de la revista viene a aparecer siete años después de su antecesor, es decir, en el segundo semestre de 1992. Nuevamente nuestras páginas fueron el reflejo de las preocupaciones de la sociedad. Una serie de artículos sobre las nuevas dinámicas económicas del país fueron publicados, en temas como la influencia del neoliberalismo (Daza, 1992) y los desafíos en la enseñanza que suponía la apertura económica (Kaffury, 1992; Leal, 1992). Al igual que en el volumen anterior, siguieron apareciendo contribuciones anónimas y otras pertenecientes al grupo de docentes de cátedra, que en ese entonces prestaban sus servicios a la Facultad.

Durante el periodo de 1993 a 1997, no fue clara la periodicidad de la publicación. Con tres números en 1993 y 1994, dos ejemplares en 1995, sin publicar en 1996 y un ejemplar en 1997, la publicación fue el reflejo de la dinámica que tenía la Facultad. En ellos los temas de actualidad se compaginaron con reflexiones de clase y documentos instituciona- 
les. Algunos artículos se ocuparon de problemáticas de comercio y café (Sánchez, 1993; Cárdenas, 1993; Leal, 1993; Sánchez, 1994), empresarios (Castañeda, 1994), apuntes de clase (Reyes, 1993, 1994; Suárez, 1995), textos orientadores para los estudiantes (Recio, 1994; Arias, 1994; Leal, 1994), y contribuciones tomadas de otras publicaciones (Prebisch, 1994; Parra-Peña, 2004; Gutiérrez, 1995). Además se incluían varios de la Facultad, algunos de la Universidad y otros de entidades externas como el Ministerio de Hacienda, Colciencias, entre otras. La línea editorial estuvo dirigida a los estudiantes como público principal de los contenidos, es por ello que las notas seleccionadas buscaban acercarles a temas de actualidad económica.

El optimismo de los primeros años de la apertura, dio paso a la crisis económica y política que afectó a Colombia durante los gobiernos de Ernesto Samper Pizano y Andrés Pastrana Arango. Una crisis financiera, que se convirtió en inmobiliaria, los efectos de la flexibilización laboral, la complicada situación de orden público ligada a la estrategia de la lucha guerrillera, la conformación de los movimientos paramilitares y la ilegitimidad de la clase política en función de los eventos de corrupción y/o debilidad, hicieron de este periodo un tiempo de ajuste, entre la modernización que exigía la nueva constitución y la reorganización de viejos poderes.

En ese entonces, la Revista empieza a ser más cuidadosa en su periodicidad, aunque no pierde la esencia con la que fue fundada. Los decanos, primero Juan López, y luego Carlos Leongomez integran algunas trasformaciones significativas. Se conforman los primeros comités editoriales, integrados fundamentalmente por los directores de los tres programas académicos de pregrado adscritos a la Facultad (Administración de Empresas, Contaduría Pública y Economía), creándose una línea de responsabilidad editorial. Además, se constituyen secciones de reflexión (economía, ecología, desarrollo, contabilidad, entre otras). Las dimensiones físicas en que se editaba la Revista también cambian, se pasa a tamaño carta, y se incluye en la tapa una franja gris con el nombre y escudo de la Universidad en la parte superior, además de una imagen alusiva a alguno de los contenidos publicados en cada número. La Revista continuó siendo el medio de comunicación de la Facultad, publicando reflexiones cortas de sus docentes y algunos documentos institucionales.

Los artículos que circulan en este periodo, reflejaron varios aspectos, por un lado cierto pesimismo frente a los resultados de la globalización y la apertura económica en nuestro país (Acosta, 1998; Mejía, 1998; Castro, 1999; Mora, 2000; Kalmanovitz, 2000; Rodríguez, 2001; Castro, 2001), la flexibilidad laboral (González, 1999), los desafíos del nuevo milenio (Marta, 1999), el orden público y el Plan Colombia (Pérez, 2001; López, 2001), temáticas de interés para las empresas (Ramírez, 2001; Borda, 2001; Ramírez \& Celis, 2001) y reflexiones educativas (Ferrucho, 2001), entre otros.

Los primeros doce años del siglo XXI han significado un periodo complejo en la historia colombiana. Un proceso social en el que diversas fuerzas han intentado transformar el Estado, a veces hacia orillas más modernas, otras veces hacia horizontes más conservadores y retardatarios. Los dos periodos presidenciales de Álvaro Uribe, incluyendo las reformas constitucionales que los hicieron posibles, el nuevo libreto de lucha contra insurgente, las repetidas tensiones en las relaciones internacionales y la llegada de capital extranjero junto a una flexibilización de las condiciones de la inversión, han sido cambios en el ámbito económico y político que representaron un nuevo devenir. En concordancia, los primeros 
años del gobierno de Juan Manuel Santos han significado continuidad, pero también transformación.

Aunado a lo anterior, en el plano educativo, muy relevantes han sido los cambios incorporados por la Ley 30 de 1993, haciendo que las instituciones de educación superior adelanten procesos de mayor competitividad, expansión de la matricula, cumplimiento de estándares y acreditación, lo que ha llevado a las universidades y escuelas a un continuo proceso de ajuste estructural. Fruto de estas presiones, entre 2004 y 2012, la Revista se constituye en un medio de divulgación científica. La Facultad entiende que ésta no puede ser únicamente un instrumento de socialización interna, sino que debe responder a las dinámicas del medio, ajustándose a la clasificación para publicaciones seriadas académicas en el marco de la política de ciencia y tecnología. La administración de Marta Eugenia Castañeda en la Facultad se propuso, entonces, hacer de la publicación uno de sus proyectos bandera, adelantando una serie de transformaciones que van a cambiar el perfil general de la publicación. De este modo, en 2005 se conformó el primer comité editorial académico, el cual se transforma en 2006 vinculando miembros reconocidos de la comunidad académica de las ciencias económicas a nivel nacional. A la par se crea el comité científico. En 2006 se nombra el primer editor, el profesor Juan Javier Saavedra, y se logra la colaboración del profesor Ernesto Ravelo Contreras como asesor experto. Los tipos de artículos a ser publicados cambian, siguiendo la línea propuesta por el Publindex. Se propone que solo se acepten documentos científicos, ya sean resultados de investigación, reflexión o revisión bibliográfica. Cambios que dan origen al subtítulo "Investigación y Reflexión" que acompaña al nombre oficial de la Revista. En este nuevo escenario, los artículos viene de diferentes instituciones, si bien en 2005 el 80\% de lo publicado tenía origen en miembros de la Universidad Militar Nueva Granada y el restante $20 \%$ en académicos de otras instituciones, para 2012 esta tendencia se ha revertido completamente.

En 2007 ingresa como editor Fredy León Paime, y en 2011 lo hace como co-editor David Camargo Mayorga. La Revista fortalece su proceso editorial, abandona su esquema de secciones, comienza a realizar procesos de arbitraje ciego de sus colaboraciones, consolida sus pautas editoriales y se propone una estrategia de visibilización de sus contenidos (proceso no sin tropiezos). Estas dinámicas no han estado exentas de dificultades, el equipo editorial ha tenido que superar con cierta disciplina la poca experticia y aprender en la práctica cómo hacer gestión editorial.

Todos estos esfuerzos tuvieron su recompensa. En 2007, la Revista fue integrada en Latindex, en 2008 en Redalyc, en el 2009 fue reconocida por Econlit (la más importante red de reconocimiento científico en ciencias económicas en el mundo). En año 2008 fue reconocida en el Publindex de Colciencias como revista científica tipo $\mathrm{C}$, luego ascendería a la categoría B en el año de 2010. También, en el 2010 la Revista fue aceptada en Scielo, a la par que fue visible en hemerotecas como Dialnet, Dotec y REPEC. Como resultado de lo anterior, son numerosos los colaboradores que en cada número desean que sus trabajos aparezcan publicados en nuestras páginas. La presencia de articulistas de diferentes partes de Iberoamérica es habitual. No es una exageración señalar, que la publicación es reconocida como una de las más importantes en las disciplinas económicas de nuestro país, lo que la ha llevado a una mejora considerable de la calidad científica de los documentos que aparecen, así como del proceso editorial que tal demanda requiere. 
Durante este periodo se han divulgado importantes aportes tanto de autores nacionales e internacionales a diferentes temáticas. Por eso, el último lustro se ha caracterizado por un proceso conjunto en el que autores, editores y evaluadores hemos trabajado para ofrecer el mejor contenido posible, en el que se valore, se aprecie y se haga amigable lo que las comunidades científicas desean difundir. Quizás nuestros procesos de arbitraje sean un poco lentos, pero son realizados con compromiso y calidad de manera constructiva, quienes han sometido sus documentos a nuestra publicación pueden dar cuenta de ello. Son tantas las temáticas y tan significativos algunos documentos, que seria imposible enumerarlos en tan corto escrito.

Los últimos dos años han reportado esfuerzos significativos para hacer de nuestra publicación una ventana competitiva en Iberoamérica. Nuevos comités, con presencia de destacados académicos de la región, acuerdos y políticas con otras publicaciones, y encuentros de editores, hacen que la gestión de la Revista se enfoque hacia nuevos rumbos. Sabemos que existen tareas pendientes y que los retos son muchos, pero consideramos que con el apoyo de todos podemos seguir creciendo. La visión que para esta publicación tenía Marta Castañeda se ha cumplido, hoy bajo una nueva decanatura, el equipo editorial mantiene ese compromiso y vocación.

Para terminar, deseamos hacer un reconocimiento a aquellos autoras y autores, provenientes tanto de nuestra institución como de instituciones amigas, quienes han confiado durante estos 20 años sus apuntes, resultados de investigación, reflexiones y opiniones. Hacemos Revista, solo si ustedes no lo permiten, permítanos seguir contribuyendo al crecimiento de las disciplinas económicas, no solo de nuestro país, sino de nuestra región.

Edison Fredy León Paime Editor

David Andrés Camargo Mayorga Co-editor

\section{Referencias}

Acosta, J. (1998). Algunas consideraciones sobre la trinidad imposible: estabilidad cambiaria, independencia monetaria y equilibrio fiscal en Colombia (privado). En: Revista Facultad de Ciencias Económicas de la Universidad Militar Nueva Granada, VI (2): 43-47.

Arias, L. (1994). Curso rápido de alta gerencia. En: Revista Facultad de Ciencias Económicas de la Universidad Militar Nueva Granada, III (5): 62-72.

Borda, P. (2000). Las funciones administrativas de planeación y control en la formulación del diagnóstico de la empresa. En: Revista Facultad de Ciencias Económicas de la Universidad Militar Nueva Granada, VIII (2): 75-83.

Cárdenas, J. (1993). Balance de las negociaciones sobre pacto cafetero y plan de retención. En: Revista Facultad de Ciencias Económicas de la Universidad Militar Nueva Granada, II (4): 5-16.
Castañeda, M. (1994). Empresarios de éxito en industrias bogotanas. En: Revista Facultad de Ciencias Económicas de la Universidad Militar Nueva Granada, III (5): 42-49.

Castro, G. (2001). Los países latinoamericanos frente a la dolarización. En: Revista Facultad de Ciencias Económicas de la Universidad Militar Nueva Granada, IX (1): 14-18.

Castro, L. (1999). Los países del tercer mundo frente a la crisis de la globalización económica y sus perspectivas. En: Revista Facultad de Ciencias Económicas de la Universidad Militar Nueva Granada, VII (2): 11-13.

Daza, A. (1992). Del liberalismo económico al neoliberalismo. En: Revista Facultad de Ciencias Económicas de la Universidad Militar Nueva Granada, II (1): 5-10.

Ferrucho, F. (2001). La efectividad personal como elemento a desarrollar en el proceso de formación que brinda la universidad. En: Revista Facultad de Ciencias Económicas de la Universidad Militar Nueva Granada, IX (1): 53-56. 
Garzón, O. (1986). Equilibrio regional y participación externa. En: Revista Facultad de Ciencias Económicas de la Universidad Militar Nueva Granada, I (1): 31-33.

González, O. (1999). Muerte laboral a los 36. En: Revista Facultad de Ciencias Económicas de la Universidad Militar Nueva Granada, VII (2): 34-37.

Gutiérrez, L. (1995). El control interno en una cultura administrativa cambiante. Traducción de: Grupo de estudios de la Oficina del Auditor General de Canadá (1992). El control interno en una cultura administrativa cambiante, Revista Opinión X (4). En: Revista Facultad de Ciencias Económicas de la Universidad Militar Nueva Granada, IV (8): 48-52.

Kaffury, M. (1992). Los programas de economía frente a la apertura económica. En: Revista Facultad de Ciencias Económicas de la Universidad Militar Nueva Granada, II (1): 11-17.

Kalmanovitz, S. (2000). El modelo anti-liberal colombiano. En: Revista Facultad de Ciencias Económicas de la Universidad Militar Nueva Granada, VIII (2): 9-17.

Leal, A. (1992). Enseñanza de la economía: una respuesta propia de la Universidad Militar. En: Revista Facultad de Ciencias Económicas de la Universidad Militar Nueva Granada, II (1): 19-22.

Leal, A. (1993). Sin pacto solo pierden los productores (Entrevista con el gerente general de Fedecafé). En: Revista Facultad de Ciencias Económicas de la Universidad Militar Nueva Granada, II (4): 17-30.

Leal, A. (1995). Nuevos programas de ciencias económicas. En: Revista Facultad de Ciencias Económicas de la Universidad Militar Nueva Granada, IV (9): 110-115.

López, D. (2001). Análisis del proceso de paz en Colombia mediante la teoría del caos. En: Revista Facultad de Ciencias Económicas de la Universidad Militar Nueva Granada, IX (1): 19-22.

Marta, L. (1999). La tecnología informática y su impacto socioeconómico en el nuevo milenio. En: Revista Facultad de Ciencias Económicas de la Universidad Militar Nueva Granada, (VII)2, 51-59.

Mejía, J. (1998). El conflicto Colombia-EE.UU de Norteamérica como entenderlo. En: Revista Facultad de Ciencias Económicas de la Universidad Militar Nueva Granada, VI (2): 67-77.

Mora, J. (2000). Apuntes sobre recesión. En: Revista Facultad de Ciencias Económicas de la Universidad Militar Nueva Granada, VIII (1): 49-54.

Parra-Peña, I. (1994). Del capitalismo periférico y el subdesarrollo. Tomado de: Parra-Peña, I. (1979). Del capitalismo periférico y el subdesarrollo, Economía Colombia, Revista Contraloría General de la República. En: Revista Facultad de Ciencias
Económicas de la Universidad Militar Nueva Granada, III (6): $135-158$.

Pérez, A. (2001). ¿Qué sabemos del plan Colombia? En: Revista Facultad de Ciencias Económicas de la Universidad Militar Nueva Granada, IX (1): 67-69.

Prebisch, R. (1994). Critica de las teorías neoclásicas del liberalismo económico. Tomado de: Prebisch, R. (1980) Critica de las teorías neoclásicas del liberalismo económico, Economía Colombia, Revista de la Contraloría General de la República. En: Revista Facultad de Ciencias Económicas de la Universidad Militar Nueva Granada, III (6): 104-134.

Ramírez, C. \& Celis, M. (2000). Habilidades gerenciales actuales y hacia el futuro. En: Revista Facultad de Ciencias Económicas de la Universidad Militar Nueva Granada, VIII (2): 84-90.

Ramírez, M. (2001). Empresa y medio ambiente. En: Revista Facultad de Ciencias Económicas de la Universidad Militar Nueva Granada, IX (2): 52-64.

Recio, A. (1994). Guía para los trabajos de grado. En: Revista Facultad de Ciencias Económicas de la Universidad Militar Nueva Granada, III (6): 173-177.

Reyes, M. (1986). Consideraciones para una administración con participación. En: Revista Facultad de Ciencias Económicas de la Universidad Militar Nueva Granada, I (1): 34-42.

Reyes, M. (1993). La administración de recursos: enfoque hacia la calidad total. En: Revista Facultad de Ciencias Económicas de la Universidad Militar Nueva Granada, II (4): 51-64.

Reyes, M. (1994). La gestión de los proyectos. En: Revista Facultad de Ciencias Económicas de la Universidad Militar Nueva Granada, III (6): 178-182.

Rodríguez, A. (1986). Consumo de alimentos en Colombia. En: Revista Facultad de Ciencias Económicas de la Universidad Militar Nueva Granada, XIV (1): 24-29.

Rodríguez, R. (2001). La globalización puede causar inestabilidad financiera a los países de América Latina. En: Revista Facultad de Ciencias Económicas de la Universidad Militar Nueva Granada, IX (1): 11-13.

Sánchez, H. (1993). Inflación, valor de la cosecha y precio interno del café: nuevo examen econométrico. En: Revista Facultad de Ciencias Económicas de la Universidad Militar Nueva Granada, II (3): 5-19.

Sánchez, H. (1994). Qué tan escaso es el mercado laboral en la zona cafetera. En: Revista Facultad de Ciencias Económicas de la Universidad Militar Nueva Granada, III (5): 10-23.

Suarez, F. (1995). Valores y objetivos de la enseñanza de la matemática. En: Revista Facultad de Ciencias Económicas de la Universidad Militar Nueva Granada, IV (9): 138-142. 